\title{
Editorial
}

The Bangladesh Journal of Bioethics is an open access journal and open to all interested and involved persons in this or related fields. The journal seeks to bring to the forefront current challenges and issues within this important sector. As a result research articles from many different countries depicting current or ongoing research, latest technological break- through, experiences from the field or literature reviews are shared with all. With the Millennium Development Goals (MDG) coming to an end in 2015, the need to assess and evaluate the progress in the 8 goals is being undertaken by different countries. At the same time Global discussions are underway on Sustainable Development beyond 2015. The Journal of Bioethics Society looks forward to submission of articles on these areas and the role played by Bioethics. The current issue presents the following articles:

Zoheb Rafique describes experiences of teaching bioethics in different institutes and degree courses at undergraduate level. Bioethics is being taught to improve the understanding of the ethical issues in the field of medicine.

Bahareh Heydari and Najmeh Razmkhah study the increasing importance of intellectual property rights (IPR) over the area of seed production, is radically transforming agricultural production relations.

Mohammad Manzoor Malik delves into the different problems of patient autonomy to euthanasia. Patient autonomy has a critical role in making decisions in medical practice and it is accepted by international conventions on health care and various national medical codes.

Muhammad Waseem Khan etal studies the different standards of care in clinical research in countries. Standard of care followed in one country may not suit other countries; the reason may involve economic conditions, certain norms, beliefs, tradition and culture of that society. The issues of conducting research in developing countries will remain and one has to try sincerely to sort out the ethical problems while conducting a research study.

Fayemi Ademola Kazeem studies the The Nijmegen Method of Case Deliberation and Clinical Decision in a Multicultural Society. Ethical case deliberation is a method of reflecting, clarifying, evaluating and making decisions about moral problems, conflicts and dilemmas in the clinical settings. The Nijmegen method helps to motivate rational decision and reasoned responsibility in healthcare through consensus building which does not attenuate moral uprightness. The Nijmegen method is part of the healthcare process and aims at improving communication among patient, family and the healthcare team as well as enriching the decision making process.

As the new Editor of this journal, I seek cooperation and involvement of all members to provide state of the art information on the important topic of Bioethics which has a tremendous impact in all spheres of our lives.

Warm Regards to all our readers and authors!

\section{Tahera Ahmed}

Consultant, Reproductive Health, UNFPA, Faculty, North South University, Former Assistant Representative, UNFPA Editor, Bangladesh Journal of Bioethics 